\title{
Acute urticaria in children: course of the disease, features of skin microbiome
}

\author{
Ekaterina Orlova, Lyudmila Smirnova, Yuri Nesvizhsky, Dmitrii Kosenkov, Elena Zykova
}

Department of Dermatology and Venereology, I. M. Sechenov First Moscow State Medical University, Sechenov University, Moscow, Russia

Adv Dermatol Allergol 2022; XXXIX (1): 164-170

DOI: https://doi.org/10.5114/ada.2022.113808

\begin{abstract}
Introduction: Quantitative and qualitative changes in the microbiome of the skin affect the emergence and course of allergic diseases, in particular, of acute urticaria.

Aim: To investigate the taxonomic composition of the skin microbiota in children with acute urticaria and to study its effect on the course of the disease.

Material and methods: In total, 75 children with diagnosed acute urticaria at the age of 7--14 years were examined. The average age of children was $10.83 \pm 0.95$, of which 44 (58.7\%) were boys, and 31 (41.3\%) were girls. The control group consisted of 30 virtually healthy children of the appropriate age, of whom $16(53.3 \%)$ were boys, and 13 (46.7\%) were girls.

Results: Regardless of the severity of the disease, the examined children suffering from acute urticaria had sensitization in history with a significant prevalence of food sensitization $(p<0.05)$. The occurrence of a severe episode of acute urticaria is associated with allergens of drug origin in $52.6 \%$ of cases and the action of unidentified triggers in $47.4 \%$ of cases. In children with acute urticaria, S. epidermidis, S. aureus, bacteria of the genus Peptococcus, and Peptostreptococcus dominated on a non-affected skin area, while for the affected skin area, the Propionibacterium, S. aureus, S. epidermidis, bacteria of the genus Peptococcus, Propionibacterium, and Peptostreptococcus were denoted as dominating.

Conclusions: High frequency of S. aureus detection on affected and non- affected skin areas in children with acute urticaria is a predictor of the disease severity.
\end{abstract}

Key words: acute urticaria in children, features of acute urticaria, sensitization and acute urticaria, skin microbiota and urticaria.

\section{Introduction}

Children's allergic diseases (AD) are quite a sensitive problem for modern medicine and the global health system being the reason for significant socio-economic expenses due to their high prevalence, which has increased dramatically in recent years [1]. The ADs have a negative impact on quality of life, increasing the frequency of concomitant pathology and the risk of death, such as in bronchial asthma [2]. Negative economic consequences of $A D$ are associated with significant direct (visit to a doctor, hospitalization, intensive care unit treatment, laboratory and instrumental diagnostics, conservative treatment, etc.) and indirect medical expenses (work and study absences, resulting in lower production volumes, worse productivity, and poor performance) [3]. The prevalence of $A D$ is quite difficult to study due to its difference within the country depending on the region, which is explained by the interaction of genetic and environmental factors [1]. Allergic diseases include several genetically heterogeneous and immunologically determined diseases such as bronchial asthma, atopic dermatitis, allergic rhinitis, food allergies, and acute urticaria (hives). Almost 700 million people worldwide suffer from respiratory ADs alone (bronchial asthma and allergic rhinitis) [4-6].

For many years, acute urticaria (AU) has been considered as nearly the most relevant problem in allergology, especially among children [7, 8]. The peak of morbidity of this pathology in children occurs in the age period from 14 to 16 years, although, over the last 5 years, a tendency

Address for correspondence: Ekaterina Orlova, Department of Dermatology and Venereology, I. M. Sechenov First Moscow State Medical University (Sechenov University), Moscow, Russia, phone: +79036181553, e-mail: orlovaeka995@rambler.ru Received: 26.08.2020, accepted: 1.12.2020. 
to higher morbidity is denoted among children of preschool and primary school age. In general, the prevalence of AU among children is 2.1-6.7\% (in Great Britain - 3.4\%, in Germany - 4.4\%, and in Denmark - 5.4\%), and among children with atopic dermatitis, the incidence rate is higher and amounts to about $16.2 \%$. This disease was observed at least once in 10-20\% of children, and in 5.4\% of cases, it was diagnosed in preschool-aged children [8]. Besides, more than half of the children with $A U$ are diagnosed with other allergic pathologies as well [9].

Infective agents, food, and medicines are among the main trigger factors for $A U$ in children [10-12]. Acute spontaneous urticaria occurs in preschool children with atopy. Infection in children is probably the most common predictor of AU compared to adults, with infection being the most frequently documented cause of this allergic pathology in children (about $40 \%$ of cases) [12, 13]. Infectious agents associated with the emergence of $A U$ include viral (rotavirus, rhinoviruses, herpes simplex virus, Epstein-Barr, hepatitis A, B, and C, and human immunodeficiency virus), bacterial (streptococcus, mycoplasma, Helicobacter pylori), and parasitic agents (protozoa, helminths). Aspirin and other non-steroidal anti-inflammatory drugs, $\beta$-lactam antibiotics, vancomycin, and opiates are the drugs most often associated with the occurrence of $\mathrm{AU}$ and cause the disease through direct degradation of mast cells. In most cases, the cause of acute urticaria is possible to establish [13].

The pathogenesis of $\mathrm{AU}$ is based on allergic reactions of immediate type, i.e., type I, less often type II and III. In such allergic reactions, allergens interact with specific antibodies-reagents of basophilic membranes and adipose cells, resulting in degranulation of these cells with the release of biologically active substances that cause typical symptoms of the disease. In particular, histamine is associated with itching, oedema, and hyperaemia on the periphery of the bladder. Histamine, cytokines, platelet activation factor incites the expansion of blood capillaries, plasma sweating, and also the migration of macrophages, neutrophils, T-lymphocytes in the area of the urticant lesion. Other mediators affecting the development of $\mathrm{AU}$ include prostaglandin D2, calcitonin binding peptide, substance $P$, and eicosanoids $[8,9]$. Non-immune mechanisms of $A U$ are much rarer and arise as a result of 1) an increase in the concentration of histamine due to its non-specific release from mast cells by the non-immune way, for example, at eating or taking some medicines; increase in the concentration of this mediator through inhibition of the enzyme activity of diamine oxidase, which directly participates in its destruction; as a result of its oversupply with certain foods (smoked food, cheeses, tuna, cod, chocolate, nuts, spinach, avocado, tomatoes, beer) and its increased synthesis in the body (e.g., in intestinal dysbiosis); 2) arachidonic acid metabolism disorders as a result of non-steroidal anti-inflammatory drug intake; 3) exces- sive acetylcholine secretion due to physical and psycho-emotional exhaustion or high-temperature action; 4) complement system activation when injecting X-raycontrast substances); and 5) bradykinin accumulation in blood, when angiotensin-converting enzyme inhibitors are taken) $[8,9,14]$.

The AU is manifested by the sudden appearance of wheals, which is associated with the influence of the trigger factor, and the duration of this condition is less than 6 weeks. Wheals do not have a certain typical localization, are accompanied by itching, and can merge in places of close contact with clothes [15]. The wheal is oedema of one of the skin layers - dermis, has a limited character, and usually disappears in 24 h. Its diameter varies from a few millimetres to several centimetres, and it is characterized by intense hyperaemia of the peripheral part and swelling of the central part. Sometimes the pathological process may extend to deeper layers of the dermis and subcutaneous adipose tissue or mucous membranes resulting in the formation of angioedema, or Quincke's oedema, for which painfulness and a longer regression of up to $72 \mathrm{~h}$ are typical. Isolated form of hives in the form of urticaria is found in $40 \%$ of cases, the Quincke's oedema in 11\% of cases [16], and the combination of urticaria and Quincke's oedema is revealed in $49 \%$ of cases [13]. Besides the local skin manifestations, children with oedema may experience increases in body temperature to febrile values, dyspepsia, abdominal and joint pain, which may lead to erroneous hospitalization in an infectious hospital [17].

The $\mathrm{AU}$ is diagnosed based on the clinical symptomatology of the disease, namely the presence of the typical urticanthropic hives described above, as well as anamnesis data relationship of its occurrence to the action of a certain agent [15]. Usually, it is sufficient for diagnosis of $\mathrm{AU}$, and there is no necessity to perform additional routine laboratory examinations. Only in case of suspicion of drug hypersensitivity or IgE-dependent mechanism of food intolerance, it is required to admit the child to the allergologist's consultation for allergy diagnostics $[13,15]$.

Treatment tactics for AU should be aimed primarily at the elimination of the triggering agent from the child's body. Of great importance is the therapeutic nutrition excluding food referred to as histamine liberators [13, 15]. Medicines of the first line include antihistamines (nonsedative), and glucocorticosteroids are recommended in case of their ineffectiveness. Noteworthy is that for symptomatic treatment of $A U$ in children, it is allowed and recommended to use only drugs with reliable evidence, which have proven clinical efficacy $[13,15,17]$.

Considering the increasing prevalence of allergic diseases among children, including AU, the issue of studying and revealing new pathogenetic links of their origin and progression is of current importance as it can improve the diagnostic algorithm and increase the efficiency of 
treatment of allergic conditions. In particular, the study of the influence of the skin microbiome on the emergence and course of AU has attracted the special attention of scientists in recent years [18-20]. Such interest in the skin microbiome is due to its extreme diversity (enrolling about 1014 taxonomic units) and absolutely unique ecosystem, the peculiarities of which depend on sex, comorbid pathology, environmental factors, a region of residence, etc. [20]. The growth of Streptococcus and/ or Propionibacterium ratio in the skin microbiome was established to correlate with the appearance of atopic dermatitis, while some Acinetobacter species, in contrast, protect the skin from allergic sensitization and inflammation and participate in maintaining the balance of Th1 and Th2 [4, 19]. The studies proved the Staphylococcus aureus, Escherichia coli, Helicobacter pylori, and Pseudomonas aeruginosa to be capable of releasing histamine from the reserves of the human body, while some taxon representatives of Acinetobacter, Serratia, Pseudomonas, Staphylococcus, Corynebacterium, and Micrococcus demonstrate histidine decarboxylating effect, which indicates that quantitative and qualitative changes of skin microbiome influence the appearance and flow of AU [21]. However, very few studies have been performed on this issue, and most of them have examined the influence of skin microbiota on the origin and course of atopic dermatitis [22, 23]. The lack of sufficient data on acute urticaria (hives) specifies the necessity of further research on this issue.

\section{Aim}

The study aimed to examine the taxonomic composition of the skin microbiota in children with acute urticaria and study its effect on the course of the mentioned disease.

\section{Material and methods}

A total of 75 children at the age of $7-14$ years with the diagnosis of acute urticaria were examined. The average age of children was $10.83 \pm 0.95$, of which 44 (58.7\%) were boys, and 31 (41.3\%) were girls. The control group consisted of 30 virtually healthy children (VHC) of the appropriate age, of whom 16 (53.3\%) were boys, and 13 (46.7\%) were girls. Surveys were conducted between 2017 and 2019 , and the number of children in this age group was 16 (53.3\%) boys and 13 (46.7\%) girls. The disease was verified following the recommendations of the European Academy of Allergology and Clinical Immunology, the European Global Allergy and Bronchial Asthma Network, the European Dermatological Forum and the World Allergy Organization (The EAACI/GA²LEN/EDF/WAO guideline for the definition, classification, diagnosis, and management of urticaria) [15].
All studies were conducted in compliance with the Convention of European Council "On Human Rights and Biomedicine" (04.04.1997), the Helsinki Declaration of the World Medical Association "On Ethical Principles for Medical Research involving Human Subjects" (19642013), and ICH Good Clinical Practice (1996).

The study included children who met the following criteria: age from 7 to 14 years, verified diagnosis of acute urticaria, voluntary informed consent to participate in the study signed by the child's parents or legal guardian.

Children, who had at least one of the following criteria, were not included in the study: age under 7 years and over 14 years, concomitant dermatological disease, history of an infectious disease suffered earlier than a month before being included in the study, intestinal infection within the last 3 months, antibiotic therapy within the last 3 months, presence of other pathology of internal organs in the acute stage, oncopathology, and mental disorders.

The level of AU severity was assessed with 0 to 3 points considering such parameters as the number of morphological elements of the rash, duration of the rash, and severity of itching. With a total score of $\leq 3$, the course of $A \cup$ was assessed as mild, AU with 4-6 scores as moderate, and with $\geq 7$ scores as severe [24].

The study of the skin microbiome in VHC was performed on the middle third of the forearm (on its inner surface), and in children with $\mathrm{AU}$ - on the affected area of the skin and on a non-affected surface symmetrical to the affected one. The material for the bacteriological study was extracted through a bacterial seal (Murray PR2015). Extracted material was sown within $1 \mathrm{~h}$ to nutrient and differential-diagnostic media produced by HiMedia Laboratories Pvt. Ltd, India, namely, HiGrome Aureus Agar Base, Endo Agar, HiGrome Enterococci Agar, HiGrome Candida Differential Agar, Blood Agar Base for facultative anaerobic bacteria, Schaedler Agar, Bacteroides Bile Esculinum Agar, and Anaerobic Agar - for non-clostridial anaerobic bacteria. After $24 \mathrm{~h}$ of incubation in a thermostat at $37^{\circ} \mathrm{C}$, the grown pathogenic colonies of microorganisms were identified by examining their morphology, tinctorial features, and biochemical properties.

\section{Statistical analysis}

The Student T-criterion and Wilcoxon non-parametric $T$-criterion were applied for statistical analysis. The differences were considered statistically significant at $p<0.05$. The $\chi^{2}$ criterion was used to numerically interpret the qualitative features and compare the detection rates of individual skin microorganisms. All statistical operations were performed using Statistica for Windows 10 Pro (Stat Soft Inc., USA) and Microsoft Excel 2013 (Microsoft, USA) software packages. 


\section{Results}

While studying the anamnesis, the food products were established as the main cause of AU development in 34 (45.3\%) children, then medicines - in 22 (29.4\%) children, and other factors, such as plant pollen, contact allergens, etc., in 7 (9.3\%) children. At that, for 12 (16\%) children, no triggering factor for $\mathrm{AU}$ has been detected. Noteworthy is that the majority of children with acute urticaria had an aggravated allergic history, i.e., 68 (90.7\%) children, of whom 46 (61.3\%) children had a history of food allergy, 29 (38.7\%) children had drug intolerance, 19 (25.3\%) children had atopic dermatitis, 8 (11.7\%) children had allergic rhinitis, and 6 (8\%) children suffered from bronchial asthma.

In considering the specifics of the disease flow, 18 (24\%) children under examination were diagnosed with the mild form of $\mathrm{AU}, 38$ (50.7\%) children had moderate $\mathrm{AU}, 19$ (25.3\%) children had severe AU. The incidence rate of moderate flow of the disease was significantly higher in comparison with the mild $(p<0.05)$ and severe $(p<0.05)$ forms of acute urticaria. No statistically significant differences in the severity of the disease between girls and boys were recorded $(p>0.05)$.

In children with a mild form, the main cause of the disease was food allergens determined in 8 (44.4\%) children, then pollen and contact allergens - in 7 (38.9\%) children, significantly less often $(p<0.05)$ compared to the triggers mentioned above. The cause of the mild OK episode was medicines - only in 3 (16.7\%) children. For all children with mild $\mathrm{AU}$, the triggering factor for the episode was possible to establish.

For moderate severity, the occurrence of the AU episode in most cases was associated with food allergens in 18 (47.4\%) children, medications in 13 (34.2\%) children, and undefined allergens in 7 (18.4\%) children.

All (100\%) children with severe AU had allergic anamnesis, while in the past, sensitization with food products prevailed significantly more often $(p<0.05)$ - in 9 (47.4\%) children. The development of severe AU episodes in the examined children was mainly related to allergens of medication origin, namely, in 10 (52.6\%) children, which is significantly more frequent than with a mild form $(p<0.05)$. The influence of unidentified triggering agents was noted in $9(47.4 \%)$ children, which is significantly more frequent than with mild $(p<0.05)$ and moderate $(p<0.05)$ degrees of severity. Severe AU in response to food allergens was not recorded in the study performed.

In addition to the main skin manifestations of $A U$ in the children included in the study, concomitant symptoms, such as general weakness, headaches, discomfort/ abdominal pain, nausea, single vomiting, and stool disorders, were also observed. Most commonly children complained of general weakness, headaches, and nausea. In children with severe disease, concomitant symptoms were reported in $100 \%$ of cases, which is significantly more frequent $(p<0.05)$ compared to moderate (concomitant symptoms were present in 24 (68.4\%) children) and mild symptoms (concomitant symptoms were present in 7 (38.9\%) children).

During the study of the species composition of skin microbiota, 11 species of microorganisms (7 facultative anaerobic (FA) and 5 non-clostridial anaerobic (NCA)) were detected in VHD and 12 species (6 FAs and 6 NCAs) were found in children with AU. The average bacteria insemination of the skin by FAs in VHD were $2.3 \pm 1.2 \mathrm{CFU} / \mathrm{sm}^{2}$, by NCAs - $1.9 \pm 0.8 \mathrm{CFU} / \mathrm{sm}^{2}$. The average bacteria insemination of the healthy skin by FAs in children with $\mathrm{AU}$ were $3.3 \pm 1.7 \mathrm{CFU} / \mathrm{sm}^{2}$, by NCAs $-1.6 \pm 0.5 \mathrm{CFU} / \mathrm{sm}^{2}$, and that of the injured skin by FAs $-3.0 \pm 1.6 \mathrm{CFU} / \mathrm{sm}^{2}$, by NCAs $-1.6 \pm 0.6 \mathrm{CFU} / \mathrm{sm}^{2}$. Among FAs, S. epidermidis was more frequently detected in 28 virtually healthy children (93.3\%), Micrococcus genus microorganisms in 18 (60\%) children (Figure 1), Peptococcus bacteria in 13 (43.3\%) children, and Peptostreptococcus in 9 (30\%)

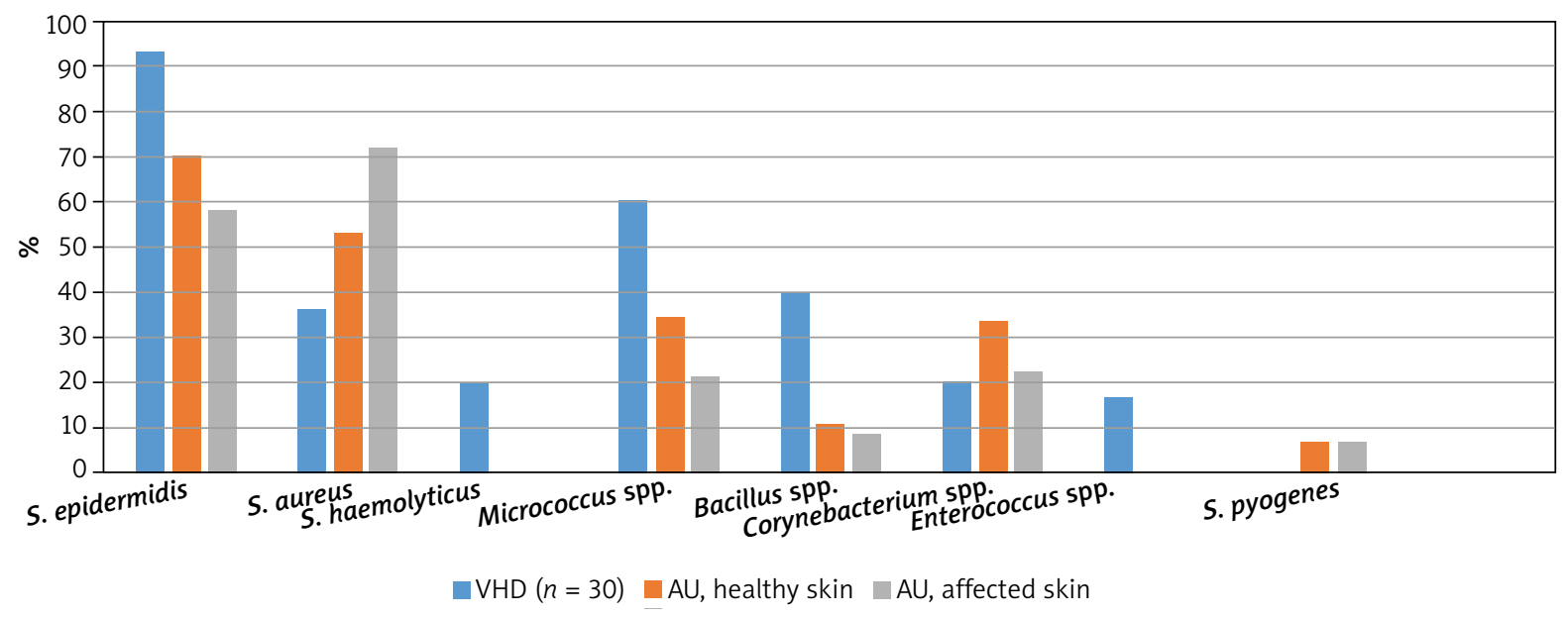

Figure 1. Emergence frequency of facultative anaerobic skin microbiotic taxons in children with acute urticaria (AU), \% 


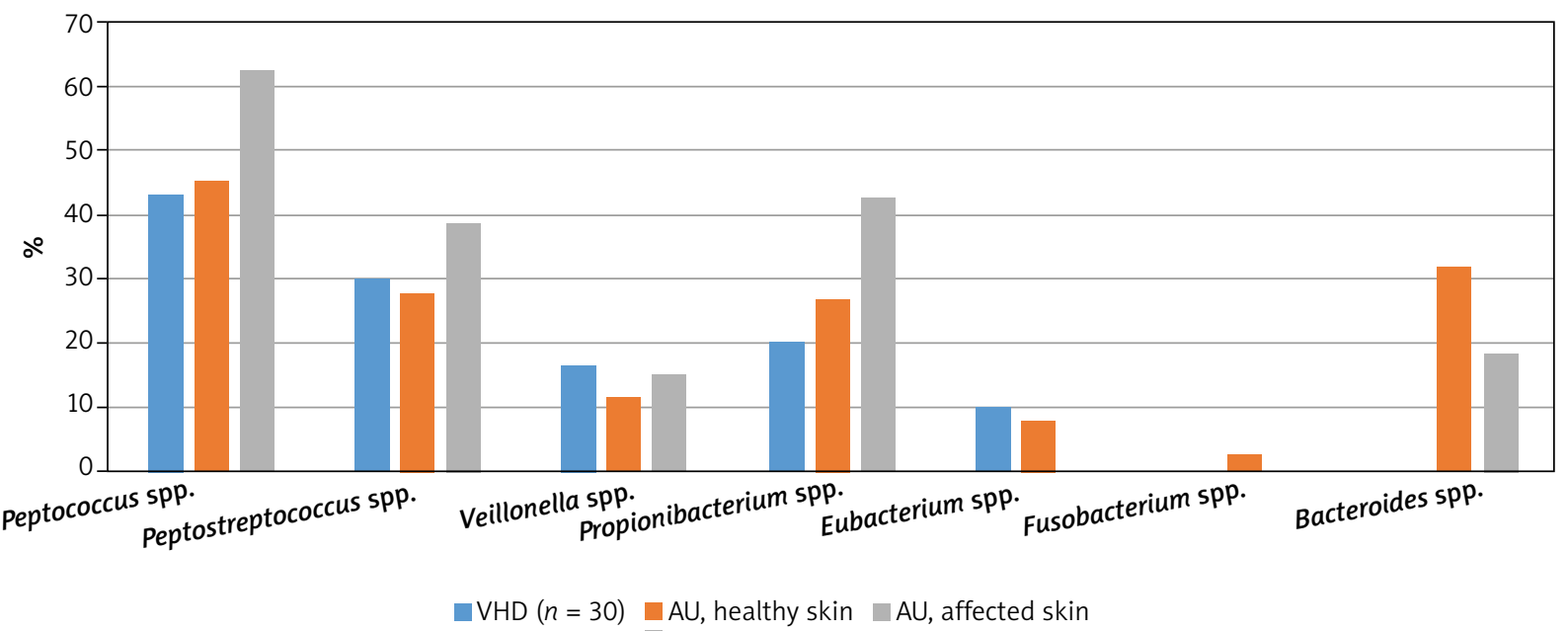

Figure 2. Emergence frequency of clostridial-anaerobic skin microbiota taxons in children with acute urticaria (AU), \%

children (Figure 2). In children with AU on a non-affected skin area, S. epidermidis dominated in 53 (70.7\%) children, S. aureus was found in 40 (53.3\%) children (Figure 1 ), Peptococcus bacteria was present in 34 (45.3\%) children, Peptostreptococcus was visible in 21 (27\%) children, and Propionibacterium was revealed in 20 (26.7\%) children (Figure 2). Those with confirmed $A U$ in the affected skin area, S. aureus dominated in 54 (72\%) children and S. epidermidis - in 44 (58.7\%) children (Figure 1). Among the NSAs, Peptococcus bacteria prevailed in 47 (62.7\%) children, Propionibacterium - in 32 (42.7\%) children, and Peptostreptococcus - in 29 (38.7\%) children (Figure 2). Thus, in children with $\mathrm{AU}, \mathrm{S}$. aureus was detected 1.96 times more frequently $(p<0.05)$ in the affected skin area as compared to VHD, and 1.45 times more frequently $(p<0.05)$ in the non-affected skin area as compared to the non-affected skin area at AU. S. epidermidis in children with $\mathrm{AU}$ was detected 1.3 times $(p<0.05)$ less frequently in the non-affected skin area compared to VHD, while in the affected area -1.6 times $(p<0.05)$ less frequently. Bacteria of genus Micrococcus were recorded 1.7 times $(p<0.05)$ and 2.8 times $(p<0.05)$ less frequently and that of genus Bacillus -4.3 times $(p<0.05)$ and 3.7 times $(p<0.05)$ less frequently in the non-affected and affected skin area, respectively. Representatives of the genus Propionibacterium were 2.1 times $(p<0.05)$ more frequently detected on the affected skin area of children with $\mathrm{AU}$ compared to VHD and 1.6 times $(p<0.05)$ more often on the affected skin area at $\mathrm{AU}$ compared to healthy areas.

The Streptococcus pyogenes was found in 5 (6.7\%) children $(p<0.05)$ with AU compared to VHD, as well as Bacteroides was detected in $14(18.7 \%)$ children $(p<0.05)$ with $\mathrm{AU}$ in the affected area of skin and 24 (32\%) children $(p<0.05)$ with AU in a healthy skin area compared to VHD. In contrast, 6 (20\%) virtually healthy children $(p<0.05)$ had Staphylococcus haemolyticus in the skin microbiome and $5(16.7 \%)$ children $(p<0.05)$ had Enterococcus genus, which was not recorded among children with acute urticaria.

The study of emergence frequency of certain microbiota taxons on the affected and non-affected areas of the skin of children with AU depending on the severity of the disease has shown that in case of the moderate $\mathrm{AU}$, bacteria of genus Bacteroides ( $p<0.05$ ) were detected on the affected area of the skin significantly more frequently in comparison with VHD against the background of the decrease of Bacillus bacteria incidence $(p<0.05)$. In the case of the severe form of the disease, the $S$. aureus bacteria $(p<0.05)$ was noticed significantly more frequently. On non-affected skin areas in children with mild AU in comparison with VHD, the bacteria of genus Propionibacterium $(p<0.05)$ were detected significantly more often, for the medium form, bacteria of genus Bacteroides $(p<0.05)$ prevailed, and for severe forms - S. aureus $(p<0.05)$. Besides, the emergence frequency of bacteria of genus Micrococcus ( $p<0.05$ ) decreased significantly in the case of a severe form of the disease.

\section{Discussion}

When studying the anamnesis and course of $A U$ in children it has been established that in $90.7 \%$ of children, a burdened allergic anamnesis for both mild and severe course of the studied disease was observed. In general, among triggering agents of $\mathrm{AU}$ episodes development in this study, food sensitization significantly prevailed accounting for $61.3 \%(p<0.05)$ of cases. At the same time, the development of a severe episode of this disease was associated with sensitization by drugs $(p<0.05)$ and unidentified triggering allergens $(p<0.05)$. Concomitant symptoms, such as general weakness, headache, nausea, vomiting, abdominal pain/discomfort, and stool disorders, were significantly more typical of the severe form 
$(100 \%$ of cases at $p<0.05)$ compared to the moderate (68.4\%) and mild (38.9\%) forms.

When examining the composition of the skin microbiota in $\mathrm{AU}$ children, a significant increase in the frequency of $S$. aureus detection $(p<0.05)$ both on the affected and non-affected skin areas was recorded compared to virtually healthy children. Besides, the emergence frequency of this microorganism increased with greater severity of the disease. It allows asserting that a higher incidence of S. aureus in children with $\mathrm{AU}$ is a predictor of the severity of this nosology.

Typical was also a significant reduction in the incidence rate of $S$. epidermidis $(p<0.05)$ in children with $\mathrm{AU}$, which is the most common microorganism on the skin of a healthy person. S. epidermidis, together with other coagulase-negative staphylococci, can release antimicrobial agents that inhibit the growth and formation of S. aureus biofilm [21]. In addition to changes in the emergence frequency of these species of staphylococcus, the variations in the incidence frequency of other skin microbiome species (genus Micrococcus, Corynebacterium, Bacillus, Propionibacterium) has been revealed, which indicates the presence of complex relationships between different taxons of skin microbiota and the need for their comprehensive and detailed study. The production of S. epidermidis and S. aureus antimicrobial substances (bacteriocins, antimicrobial peptides) incites a decrease in the relative number of Micrococcus, Bacillus, and S. haemolyticus bacteria during the $\mathrm{AU}$ episode [23, 25].

The data obtained concerning the main triggering factors of AU episodes development and its flow features are generally comparable with the results of other similar studies [17, 24]. However, more research in the literature is devoted to the study of skin microbiome features in atopic dermatitis enrolling both adults and children [22, 26]. In particular, a study involving 128 people aged 2-62 years revealed the predominance of healthy individuals of the bacteria of genera Staphylococcus, Propionibacterium, Corynebacterium, and Streptococcus on the skin microflora, and, S. aureus, Propionibacterium, and Peptococcus on the skin of patients with atopic dermatitis [26], which is consistent with the results of this research. A similar study was conducted in Russia with $61 \mathrm{AU}$ children at the age of 3-12 years. It has been established that the severe flow of $\mathrm{AU}$ is associated with medication sensitization and increased frequency of $S$. aureus detection on the affected skin area in AU children [24], which also corresponds to results of this study.

\section{Conclusions}

Thus, the results obtained in this study showed that, regardless of the severity of the acute urticaria flow, the surveyed children suffering from this disease had sensitization in their history, namely, $61.3 \%$ of children - food allergy, $38.7 \%$ - medication intolerance, $25.3 \%$ - atopic dermatitis, $11.7 \%$ - allergic rhinitis, and $8 \%$ - bronchial asthma. At that, food sensitization prevailed $(p<0.05)$. The occurrence of a severe episode of acute urticaria is associated with allergens of medication origin (in $52.6 \%$ of cases) and the action of unidentified triggers (in $47.4 \%$ of cases), which is significantly more frequent in comparison with a mild form of the disease $(p<0.05)$. In children with acute urticaria, S. epidermidis dominated in a non-affected skin area (in $70.7 \%$ of cases), while S. epidermidis. S. aureus (in 53.3\%), bacteria of the genus Peptococcus (in 45.3\%), Peptostreptococcus (in $27 \%$ ), Propionibacterium (in $26.7 \%$ ), S. aureus (in $72 \%$ ), S. epidermidis (in 58.7\%), bacteria of the genus Peptococcus (in 62.7\%), Propionibacterium (in 42.7\%) and Peptostreptococcus (in 38.7\%) prevailed on the affected area. In children with AU, S. aureus was detected 1.96 times more frequently $(p<0.05)$ in the affected skin area as compared to VHD, and 1.45 times more frequently $(p<0.05)$ in the non-affected skin area as compared to the non-affected skin area at AU. S. epidermidis in children with $\mathrm{AU}$ was detected 1.3 times $(p<0.05)$ less frequently in the non-affected skin area compared to VHD, while in the affected area -1.6 times $(p<0.05)$ less frequently. High frequency of $S$. aureus detection on the affected and non-affected skin areas in children suffering from acute urticaria can be considered as a predictor of the disease severity.

Prospects for further research: a prospect for further research is to examine the efficacy of probiotic use in children with severe and severe acute hives.

\section{Conflict of interest}

The authors declare no conflict of interest.

\section{References}

1. Doğruel D, Bingöl G, Altıntaş DU, et al. The trend of change of allergic diseases over the years: three repeated surveys from 1994 to 2014. Int Arch Allergy Immunol 2017; 173: 178-82.

2. Wang IJ, Tung TH, Tang CS, Zhao ZH. Allergens, air pollutants, and childhood allergic diseases. Int J Hyg Envir Heal 2016; 219: 66-71.

3. Hendaus MA, Jomha FA, Ehlayel M. Allergic diseases among children: nutritional prevention and intervention. Ther Clin Risk Manag 2016; 12: 361-72.

4. Pascal M, Perez-Gordo M, Caballero T, et al. Microbiome and allergic diseases. Front Immunol 2018; 9: 1584.

5. Zhang Y, Zhang L. Increasing prevalence of allergic rhinitis in China. Allergy Asthma Immunol Res 2019; 11: 156-69.

6. Kansen HM, Le TM, Uiterwaal C, et al. Prevalence and predictors of uncontrolled asthma in children referred for asthma and other atopic diseases. J Asthma Allergy 2020; 13: 67-75.

7. Ben-Shoshan M, Grattan CE. Management of pediatric urticaria with review of the literature on chronic spontaneous urticaria in children. J Allergy Clin Immunol 2018; 6: 1152-61.

8. Kudryavtseva AV, Neskorodova KA, Staubach P. Urticaria in children and adolescents: an updated review of the pathogenesis and management. Pediatr Allerg Imm 2018; 30: 17-24. 
9. Talarico V, Marseglia GL, Lanari M, et al. Pediatric urticaria in the Emergency Department: epidemiological characteristics and predictive factors for its persistence in children. Eur Ann Allergy Clin Immunol 2021; 53: 80-5.

10. Bedolla-Barajas M, Kestler-Gramajo A, Alcalá-Padilla G, Morales-Romero J. Prevalence of oral allergy syndrome in children with allergic diseases. Allergol Immunopath 2017; 45: 127-33.

11. Lee SJ, Ha EK, Jee HM, et al. Prevalence and risk factors of urticaria with a focus on chronic urticaria in children. Allergy Asthma Immunol Res 2017; 9: 212-9.

12. Shin M, Lee S. Prevalence and causes of childhood urticaria. Allergy Asthma Immunol Res 2017; 9: 189-90.

13. Schaefer P. Acute and chronic urticaria: evaluation and treatment. Am Fam Physician 2017; 95: 717-24.

14. Choi YJ, Yoon JM, Chang YS, Oh JW. Sensitization to component antigens in acute idiopathic urticaria in children. Allergy Asthma Immunol Res 2017; 5: 331-5.

15. Zuberbier T, Aberer W, Asero R, et al. The EAACI/GA2LEN/ EDF/WAO guideline for the definition, classification, diagnosis and management of urticaria. Allergy 2018; 73: 1393-414.

16. Veronez CL, Grumach AS. Angioedema without urticaria. Curr Opin Allergy Clin Immunol 2020; 20: 253-60.

17. Losappio L, Heffler E, Bussolino C, et al. Acute urticaria presenting in the emergency room of a general hospital. Eur J Intern Med 2014; 25: 147-50.

18. Johnson CC, Ownby DR. The infant gut bacterial microbiota and risk of pediatric asthma and allergic diseases. Transl Res 2017; 179: 60-70.

19. Musso P, Chiappini E, Bernardini R. Human microbiome and allergic diseases in children: pathogenetic role and therapeutic options. Curr Pediatr Rev 2019; 16: 89-94.

20. Nance CL, Deniskin R, Diaz VC, et al. The role of the microbiome in food allergy: a review. Children 2020; 7: E50.

21. Kudagammana ST, Vidanapathirana GU. Human microbiome and its role in paediatric allergic disease. Sri Lanka J Child Health 2020; 49: 102-7.

22. Brunner PM, Leung DYM, Guttman-Yassky E. Immunologic, microbial, and epithelial interactions in atopic dermatitis. Ann Allergy Asthma Immunol 2018; 120: 34-41.

23. Kong HH, Oh J, Deming C, et al. Temporal shifts in the skin microbiome associated with disease flares and treatment in children with atopic dermatitis. Genome Res 2012; 22: 850-9.

24. Posevina AN, Lebedenko AA, Naboka YL, et al. Skin microbiota of children with acute urticaria. Vestnik Nacional'nogo mediko-hirurgicheskogo Centra im. N.I. Pirogova 2016; 11: 56-8.

25. Reiger M, Traidl-Hoffmann C, Neumann AU. The skin microbiome as a clinical biomarker in atopic eczema: promises, navigation, and pitfalls. J Allergy Clin Immunol 2020; 145: 93-6.

26. Shi B, Bangayan NJ, Curd E, et al. The skin microbiome is different in pediatric versus adult atopic dermatitis. J Allergy Clin Immunol 2016; 138: 1233-6. 